\title{
Produzindo conhecimento sobre família: a contribuição da enfermagem do Sul do Brasil
}

\author{
Producing knowledge about the family: a contribution from nursing in the south of Brazil \\ Produciendo conocimiento sobre la familia: la contribución de la enfermería del Sur de Brasil \\ Sonia Silva Marcon ${ }^{1}$, Maria Angélica Pagliarini Waidman², Maria \\ das Neves Decesaro ${ }^{3}$, Marcia Maria Marino Arêas ${ }^{4}$
}

\begin{abstract}
RESUMO
Objetivos: Estudo descritivo-exploratório que identificou e caracterizou os aspectos teóricos e metodológicos de estudos de pós-graduação em enfermagem que tiveram a família como tema norteador. Métodos: Foram analisadas 45 investigações. Os dados foram coletados no período de março a junho de 2002, junto aos autores dos estudos, por meio de instrumento semi-estruturado. Resultados: Os resultados revelaram que $66,6 \%$ dos estudos foram desenvolvidos na Universidade Federal de Santa Catarina e que a maior proporção foi defendida no ano 2000 (26,7\%), utilizando como referencial teórico-metodológico as teorias de enfermagem (40\%). Em 68,9\% dos estudos os dados foram coletados no domićlio. O cuidado à família e a convivência familiar constituíram temas de, respectivamente, 44,6\% e 34,0\% dos estudos, cujos objetivos foram agrupados em seis categorias.Conclusões: O interesse por esta temática vem aumentando; grande parte dos estudos ainda são de caráter descritivo-exploratório e apresentam objetivos amplos; os aspectos abordados normalmente emergem do cotidiano profissional e, embora a maioria dos estudos tenham utilizado o domicílio como cenário da investigação, este foco tem sido ampliado.
\end{abstract}

Descritores: Pesquisa; Enfermagem; Família.

\begin{abstract}
Objectives:This is a descriptive and exploratory research, having the family as the guiding theme, which aimed to identify and characterize theoretical and methodological aspects of research conducted during graduate studies at Brazilian nursing schools. Methods: The sample consisted of 45 studies. Data were collected with a semi-structured instrument from March to June, 2002. Results: The results revealed that the majority of studies $(66.6 \%)$ were conducted at Federal University of Santa Catarina, a great part of them $(26.7 \%)$ were defended in 2000 and used nursing theories as theoretical or conceptual framework $(40.0 \%)$. The majority of the data $(68.9 \%)$ were collected in the family homes. Family care (44.6\%) and the relationship among family members (34.0\%) were the themes of most studies. These themes were grouped into six categories Conclusions: Although the family as a theme for research has been used in many studies over the years, most research has focused on descriptive and exploratory investigations. These studies normally addressed broad purposes and used approaches emerging from researcher' professional experience. Most studies have used family homes as the background for the investigation.
\end{abstract}

Keywords: Knowledge; Nursing; Family.

\section{RESUMEN}

Objetivos: Estudio descriptivo-exploratorio que identificó y caracterizó los aspectos teóricos y metodológicos de estudios de post grado en enfermería que tuvieron a la familia como tema orientador. Métodos: Fueron analizadas 45 investigaciones. Los datos fueron recolectados en el período de marzo a junio del 2002, junto a los autores de los estudios, por medio de un instrumento semi-estructurado. Resultados: Los resultados revelaron que el 66,6\% de los estudios fueron desarrollados en la Universidad Federal de Santa Catarina y que la mayor proporción fue sustentada en el año 2000 (26,7\%), utilizando como referencial teórico-metodológico las teorías de enfermería (40\%). En el $68,9 \%$ de los estudios los datos fueron recolectados en el domicilio. El cuidado a la familia y la convivencia familiar constituyeron temas de, respectivamente, $44,6 \%$ y $34,0 \%$ de los estudios, cuyos objetivos fueron agrupados en categorías. Conclusiones: el interés por esta temática viene aumentando; gran parte de los estudios aún son de carácter descriptivo-exploratorio y presentan objetivos amplios; los aspectos abordados normalmente emergen del cotidiano profesional y, a pesar que la mayoría de los estudios hayan utilizado el domicilio como escenario de la investigación, este foco ha sido ampliado.

Descriptores: Investigación; Enfermería; Familia.

\footnotetext{
1 Doutora em Enfermagem. Professora da Universidade Estadual de Maringá (PR), Brasil.

${ }^{2}$ Doutora em Enfermagem. Professora da Universidade Estadual de Maringá (PR), Brasil.

${ }^{3}$ Doutoranda em Enfermagem da Escola de Enfermagem da Universidade de São Paulo - Ribeirão Preto (SP), Brasil
} 


\section{INTRODUÇÃO}

Nos últimos anos e em diferentes campos do conhecimento tem sido observado um aumento crescente da produção científica na área da família, resultante de um interesse que ultrapassa as fronteiras das disciplinas, ao envolver profissionais, pesquisadores, organizações governamentais e não governamentais. No âmbito da saúde, mais especificamente na enfermagem e na medicina, a partir de 1980 intensificou-se o interesse por esta temática em diversas regiões do mundo, sendo que no Brasil ele teve início na segunda metade dessa mesma década, principalmente na enfermagem. A partir de meados da década de 1990, com a implantação do Programa de Saúde da Família pelo Ministério da Saúde, passou-se a preconizar e incentivar a adoção, por parte das equipes de saúde, de estratégias e ações que valorizem a integração com a população e a promoção da saúde, como forma de garantir melhorias efetivas nos níveis de saúde da população.

Com isto, abriram-se novas perspectivas para a atuação dos profissionais - especialmente médicos e enfermeiros - nos serviços de saúde, e se tornou necessário promover mudanças na formação desses profissionais, assim como o seu aperfeiçoamento, por meio de educação permanente e da busca por cursos de especialização e residência com uma abordagem multidisciplinar.

$\mathrm{Na}$ área da enfermagem, as mudanças na formação procuraram dar conta de uma intervenção que reconheça a existência de dois sistemas de saúde, o profissional e o popular, e que também compreenda as diferenças culturais e procure preservar o cuidado já conhecido e utilizado pelo cliente. Isto possibilita um cuidado mais congruente com as reais necessidades do indivíduo e sua família ${ }^{(1)}$.

Com as mudanças na forma de ensinar enfermagem abrem-se novas perspectivas também para o exercício dessa profissão, que passa a valorizar não só a inserção da família como recurso de assistência ao indivíduo, mas também a própria família como objeto de trabalho e de investigação. Os resultados dessas mudanças têm sido observados em diferentes segmentos, inclusive em relação à produção do conhecimento.

No início, os estudos eram realizados quase exclusivamente no âmbito acadêmico, tendo-se como preocupação desenvolver instrumentos para assistir adequadamente a família, enquanto unidade de cuidado.

Atualmente, já é possível perceber uma utilização do conhecimento produzido na academia, de forma que a família tem constituído tema freqüente nas comunicações feitas por profissionais de serviço.

A investigação de aspectos da produção científica da enfermagem brasileira em relação à temática família nos resumos dos trabalhos apresentados nos congressos brasileiros de enfermagem no período de 1985 a 1996 levou à constatação de que 4,9\% deles estavam relacionados ao tema família; e mais: que o interesse nesta área vinha aumentando lenta, porém regularmente, de modo especial nos estados de São Paulo, Rio de Janeiro, Ceará e Santa Catarina ${ }^{(2)}$. Constatou-se, ainda, que a Região Sudeste foi responsável por quase metade das comunicações (42,8\%), seguida das regiões Sul (16,8\%) e Norte $(11,6 \%)$. Isto parece estar relacionado à concentração dos cursos de graduação e pós-graduação de enfermagem e de profissionais em atividade nestas regiões.

Considerando estes aspectos, em 2002 buscou-se identificar como estava a produção sobre esta temática nos cursos de pós-graduação, por meio de um levantamento de toda a produção científica de enfermagem cadastrada no Centro de Estudos e Pesquisa em Enfermagem- CEPEn no período de 1979 a $2001^{(3)}$,e constatou-se que foi somente a partir da década de 1990 que houve um incremento na produção de estudos com família no Brasil, e, que até então, apenas seis instituições eram responsáveis por $93 \%$ dos estudos desenvolvidos com esta temática pela enfermagem brasileira na pósgraduação (Universidade Federal de Santa Catarina UFSC, Escola de Enfermagem da USP - SP, Escola de Enfermagem Anna Nery - EEAN, Universidade Federal do Ceará - UFCe e Universidade Federal da Paraíba UFPa), sendo que a UFSC foi responsável por $43 \%$ dos trabalhos quando, considerados apenas aqueles cadastrados no termo identificador família, e por $33,6 \%$ quando se consideraram trabalhos cadastrados também em outros termos identificadores. Isto revela que uma parcela considerável das investigações de pós-graduação em enfermagem com famílias foi desenvolvida em programas localizados na região sul do país.

Ante o exposto, o presente trabalho tem por objetivo conhecer os aspectos teóricos e metodológicos dos estudos de pós-graduação que tiveram a família como tema norteador.

\section{MÉTODOS}

Estudo exploratório descritivo desenvolvido junto a 43 informantes - enfermeiros que tinham em comum o fato de terem desenvolvido seus estudos de mestrado e/ ou doutorado abordando a temática família. Os informantes se manifestaram em relação a 45 a partir das respostas apresentadas pelos informantes, não tendo sido consultados os estudos originais. Para a coleta de dados foi utilizado um questionário semi-estruturado, constituído de 28 questões distribuídas em cinco partes: dados de identificação, caracterização do estudo, referencial teórico, referencial metodológico e resultados. O instrumento de coleta de dados foi avaliado por três peritos de diferentes áreas (enfermagem, psicologia e serviço social) com 
experiência no trabalho com famílias nos âmbitos do ensino de pós-graduação e da pesquisa .

O desenvolvimento do estudo levou em consideração os preceitos éticos disciplinados pela Resolução 196/96 do Conselho Nacional de Saúde ${ }^{(4)}$. A solicitação de participação no estudo foi enviada aos autores, juntamente com o instrumento de coleta de dados, via Internet.

Os dados quantitativos foram analisados com o auxilio da estatística descritiva e estão apresentados em termos percentuais. Para a análise dos dados de natureza qualitativa, foi dada ênfase ao conteúdo das mensagens expressas, numa tentativa de identificar e categorizar aspectos relevantes das investigações com família apontados pelos próprios autores. Para tanto, inicialmente procedeu-se ao agrupamento de todas as respostas de uma mesma questão; e após leitura exaustiva do conjunto de dados de cada uma delas, foi feita a análise temática e em seguida a categorização ${ }^{(5)}$.

\section{RESULTADOS E DISCUSSÃO}

Das 45 investigações em estudo, 33 são dissertações de mestrado e 12 são teses de doutorado. A maior freqüência de defesa de estudos com família ocorreu na UFSC (66,6\%) e no ano 2000 (26,7\%). Dos 30 estudos defendidos na UFSC, $22(70 \%)$ foram de mestrado, e destes, 8 foram desenvolvidos em um dos 3 cursos ofertados pela Repensul - Rede Pós-graduação em Enfermagem da Região Sul.

TABELA 1 - Distribuição do n. ${ }^{\circ}$ e \% das investigações, segundo o ano de defesa do estudo. Maringá - PR, 2002

\begin{tabular}{lcr}
\hline Ano de Defesa & Número & $\mathbf{\%}$ \\
\hline Antes de 1990 & 02 & 4,4 \\
De 1991 até 1995 & 05 & 11,2 \\
1996 & 03 & 6,7 \\
1997 & 03 & 6,7 \\
1998 & 07 & 15,5 \\
1999 & 04 & 8,9 \\
2000 & 12 & 26,7 \\
2001 & 07 & 15,5 \\
2002 & 02 & 4,4 \\
\hline Total & 45 & 100,0 \\
\hline
\end{tabular}

Ressalte-se que no âmbito nacional, ou seja, quando considerados os estudos realizados em todos os cursos de pós-graduação em enfermagem no Brasil, constatouse que também foi no ano 2000 que ocorreu a maior freqüência $(37 \%)$ de defesas de estudos com esta temática ${ }^{(3)}$.

O fato de a maioria dos estudos ter sido desenvolvida na UFSC atribui característica singular ao conjunto das investigações em estudo, pois nessa instituição a ênfase das dissertações de mestrado está em desenvolver pesquisas práticas, o que tem por propósito promover mudanças na realidade, mediante a implementação de uma metodologia de assistência fundamentada em referencial teórico, denominada de pesquisa convergente assistencial $^{(6)}$. No total, 15 investigações $(33,3 \%)$ foram classificadas por seus autores como pertencentes a esta modalidade de estudo. O mesmo percentual $(33,3 \%)$ foi observado em relação aos estudos identificados como exploratório-descritivos; e dos demais, 9 (20\%) foram identificados como exploratórios e $6(13,4 \%)$ como descritivos.

Enquanto a maioria dos estudos de família desenvolvidos por enfermeiros da Região Sul do Brasil são de natureza qualitativa (70\%), um estudo que analisou 76 artigos publicados em cinco periódicos de enfermagem, no período de 1986 a 1993, nos Estados Unidos $^{(7)}$, constatou que em sua maioria eles eram quantitativos (apenas cinco qualitativos) e de caráter descritivo ou correlacional. Com relação a este aspecto, observou-se, na presente investigação, que grande parte dos estudos é do tipo exploratório-descritivo (33,3\%), modalidade que se mostra adequada ao propósito de desvelar características das famílias, dos grupos e serviços de atendimento e, portanto, apropriada para o uso na enfermagem, que se mostra preocupada em estabelecer um diagnóstico da situação para só então propor mudanças na realidade estudada.

No que se refere ao local do estudo, observamos na tabela 2 que a maioria deles $(68,9 \%)$ foi desenvolvida no contexto do domicílio. O lar é o local social natural, e por esta razão constitui um contexto mais rico para estudos qualitativos da vida familiar ${ }^{(8)}$. Outra vantagem apontada é o fato de este cenário ser um local mais confortável e descontraído, onde um maior número de membros da família pode participar da pesquisa. Já as desvantagens apontadas relacionam-se aos custos financeiros, ao gasto de tempo e à necessidade de, em alguns casos, ser necessária a presença de um número maior de pesquisadores ${ }^{(8)}$.

Nossa experiência em trabalhar com famílias tem mostrado lacunas importantes relacionadas com a metodologia de assistência de enfermagem no domicilio. No trabalho com a família faz-se necessária a abordagem interdisciplinar, além da adoção de estratégias alternativas com trabalhos de grupo, oficinas de artesanato, entre $\operatorname{outros}^{(9)}$. Acreditamos que as lacunas existentes têm impulsionado pesquisadores a desenvolver seus estudos visando ao atendimento dessas dificuldades, o que provavelmente justifica o fato de a maioria dos estudos ter utilizado como cenário para a coleta de dados o domicílio, embora alguns tenham associado a este o hospital, a comunidade e o ambulatório. Ressalte-se, ainda, que os profissionais enfermeiros têm atuado de maneira muito próxima à família e, talvez por isso, buscam nos estudos as respostas para os questionamentos que 
emergem de suas atividades cotidianas.

Tabela 2 - Distribuição do n. ${ }^{\circ}$ e \% das investigações, segundo o local de coleta de dados. Maringá - PR, 2002

\begin{tabular}{lcr}
\hline Local de coleta de dados & $\mathbf{N}^{\mathbf{0}}$ & $\mathbf{\%}$ \\
\hline Só no domić́lio & 18 & 40,0 \\
No domić́lio e hospital & 05 & 11,1 \\
No domićlio e comunidade & 03 & 6,7 \\
No domićlio e ambulatório/UBS & 05 & 11,1 \\
Só na Instituição de saúde & 12 & 26,7 \\
Comunidade & 01 & 2,2 \\
Pesquisa bibliográfica & 01 & 2,2 \\
\hline Total & 45 & 100,0 \\
\hline
\end{tabular}

O uso do referencial teórico como guia no desenvolvimento e interpretação dos resultados de um estudo permite ao pesquisador direcionar sua lente para uma compreensão sistematizada dos fatos ou fenômenos em estudo, uma vez que a adoção de abordagens teóricas contribui para fortalecer a construção do conhecimento para melhor intervir.

Quanto à utilização de referencial teóricometodológico, observamos inicialmente que seis informantes não se manifestaram, não sendo possível saber se foi ou não utilizado algum referencial. Os autores de 18 estudos revelaram ter utilizado como referencial teorias de enfermagem, sendo a de Leininger - Teoria Trancultural - a mais freqüente (nove estudos). Acreditamos que isto se deva à forma de a autora propor a compreensão dos sistemas leigo e profissional de cuidado, e principalmente ao fato de sua abordagem valorizar os aspectos culturais, sendo que o foco central da teoria leva em consideração as crenças, valores e costumes das famílias. Assim, respeitar seus princípios, transportando-os para a prestação da assistência ou cuidado, permite uma maior aproximação com o grupo familiar, possibilitando que a pesquisa seja desenvolvida com menos resistência. As demais teorias de enfermagem utilizadas são de autoras como Watson (três), Paterson e Zderad (duas), Travelbee (duas), King (uma) e Nigthingale (uma), que valorizam a interação e a relação interpessoal. Consideramos que o referencial humanístico discutido por estas teoristas, ao abordarem as relações interpessoais ou transpessoais, satisfaz, pelo menos em parte, a necessidade de interação que existe dentro do contexto familiar, tanto em relação ao indivíduo ou à família, no que tange a sua forma de viver, quanto em relação ao profissional que pretenda compreender a vida familiar.

Observamos, também, que 21 estudos utilizaram referenciais teóricos de outras áreas do conhecimento, porém muito utilizados na enfermagem, tais como o Interacionismo Simbólico (nove estudos), a Fenomenologia (quatro estudos) a Teoria Sistêmica (três estudos) e a Representação Social (em dois estudos). Acreditamos que a escolha destes referenciais tenha ocorrido em função da forma como eles propõem sua utilização e dos aspectos considerados relevantes na sua abordagem, o que, por sua vez, possibilita a análise de relações e melhor compreensão da realidade familiar. Os referenciais próprios de família abordam a vida familiar e ao mesmo tempo descrevem o conjunto das interações sociais, demonstrando assim toda a sua apropriação para o trabalho com famílias no ensino, pesquisa e assistência. Já os da enfermagem têm como característica a possibilidade de serem aplicados na prática, e nestes casos, têm-se mostrado mais apropriados para a compreensão de outros elementos do processo de viver das famílias, e também para subsidiar as intervenções em eventos situacionais ou críticos da vida familiar.

Em relação ao tema do estudo, é preciso considerar que em qualquer tipo de pesquisa a escolha do assunto deverá refletir a relevância contemporânea e humana, além de ser dirigida para a prática, isto é, constituir algo que mereça ser aprofundado ${ }^{(10)}$. Assim, constatamos que o cuidado à família ou ao familiar doente foi o tema de estudo mais freqüente, seguido da convivência familiar (Tabela 3). O fato de o cuidado permear grande parte dos estudos deve-se à concepção dos profissionais da enfermagem de que este constitui o foco central da disciplina. Se isto for utilizado também na prática com família, de acordo com o referencial teórico escolhido, é importante conhecer e compartilhar a concepção de cuidado construída pela família, para só então cuidar da mesma, pois já é consenso que o trabalho com famílias é um compartilhar de saberes.

Tabela 3 - Distribuição do n. ${ }^{\circ}$ \% \% das investigações, segundo o tema dos estudos. Maringá - PR, 2002

\begin{tabular}{lcc}
\hline Tema abordado & $\mathbf{N}^{\mathbf{0}}$ & $\mathbf{0}$ \\
\hline Cuidado á família/familiar & 22 & 46,8 \\
Convivência familiar & 17 & 36,1 \\
Crenças e práticas da família & 02 & 4,3 \\
Educação para a saúde & 02 & 4,3 \\
Metodologia & 02 & 4,3 \\
Violência familiar & 01 & 2,1 \\
Reprodução humana & 01 & 2,1 \\
\hline Total & $\mathbf{4 7 *}$ & $\mathbf{1 0 0 , 0}$ \\
\hline
\end{tabular}

* Duas investigações apresentavam mais de uma temática

No que se refere à convivência familiar, é importante destacar que esta quase sempre foi focalizada em sua interface com a saúde e a doença. No entanto, é na convivência familiar que se percebem as necessidades diferenciadas da família, as quais estão diretamente ligadas ao momento do ciclo de vida que elas vivenciam, à cultura, à etnia e à classe social a que pertencem ${ }^{(10)}$. Ressaltese que o profissional deve estar atento para o fato de 
que, embora as famílias e suas necessidades guardem relação com o ciclo de vida que elas vivenciam, ainda assim as necessidades são diferentes em sua essência.

$\mathrm{Na}$ tabela 3, também chama a atenção a pouca freqüência de alguns temas que consideramos relevantes, e dentre eles destacamos a educação para a saúde e a violência familiar, uma vez eles permeiam a prática da enfermagem junto à família. A educação para a saúde com famílias deve envolver uma proposta de parceria na qual a família participe ativamente de programas de intervenção que visem à promoção da saúde da família como um todo e do bem-estar de cada um de seus membros ${ }^{(11,12)}$.

Quanto à violência familiar, destacamos sua associação a diversos fatores, pois a família, fazendo parte da sociedade, influencia esta e é por ela influenciada, podendo manifestar nesta relação um de seus fenômenos mais críticos - a violência. Cabe destacar que a família, ao vivenciar a situação de violência, além de abandonar seu lado protetor e de cuidado, enquanto unidade, passa a apresentar vulnerabilidade, necessitando de assistência com caráter interdisciplinar ${ }^{(14)}$.

A violência familiar constitui importante problema de saúde pública, discutido e presente em todas as classes sociais, devendo ser enfrentado pelos profissionais de saúde. No entanto, nos estudos analisados, este tema foi abordado em uma única investigação, desenvolvida há mais de uma década. Isto nos leva a questionar se o fato de os enfermeiros da Região Sul do País que trabalham com famílias não se aventurarem por este caminho não está relacionado a um receio/dificuldade pessoal em lidar com esta questão.

Em relação aos temas abordados nos estudos, as pesquisas de enfermagem com famílias apresentam uma grande diversidade nos aspectos antropológicos, psicológicos, sociais, políticos e de saúde ${ }^{(15)}$. Isso nos remete a uma busca pela adaptação bem-sucedida em mudanças nas políticas públicas de saúde e família.

Esta diversidade na abordagem da temática também tem sido identificada em nosso meio tanto nas publicações dessa natureza quanto em eventos de caráter interdisciplinar sobre pesquisa com família. De forma geral, os trabalhos com famílias são classificados em quatro grandes temáticas: relações familiares; cuidado da família e a família no processo saúde doença; contexto social e família; e formação profissional e metodologias de atendimento à família ${ }^{(10)}$.

Quando se busca identificar os objetivos que os profissionais procuraram atingir no desenvolvimento do estudo, observamos que esses objetivos podem ser agrupados em seis categorias, sendo que "Compreender como a família vivencia determinadas situações" englobou o maior número de estudos $(30,4 \%)$. Compreender é aqui entendido como desvelar, de forma integral, os processos/experiências vivenciados pelos familiares em situações em que um dos membros se apresenta doente e fragilizado. Para tanto, observou-se que os autores buscaram entender não apenas como a família vivencia a facticidade da doença e a necessidade do cuidado, mas também tudo aquilo que envolve o contexto e o viver familiar, ou seja, as crenças, valores, percepções, sentimentos experienciados, comportamentos manifestos. Além disso, tentaram compreender como ocorrem as interações familiares durante a trajetória em que estão convivendo com o risco de vida.

A segunda categoria, "Conhecer como a família cuida e quais suas necessidades de saúde" (23,9\%), engloba estudos que se propuseram conhecer o cotidiano, costumes, comportamentos, crenças, necessidades da família e do portador da doença, possibilidades e limitações para a prática do cuidado realizado no domicílio. Incluiu ainda a identificar a realidade do sistema de cuidado familiar, a rede social de suporte das famílias e as representações sociais de cuidadores sobre o cuidado assumido por eles, bem como a sobrecarga decorrente desta atividade.

A terceira categoria foi "Implementar uma metodologia assistencial" (16,3\%) que priorize a valorização do cuidado humanizado, por meio do desenvolvimento de um processo educativo-participativo que integre a família, ora com os discentes ora com os docentes ou a equipe assistencial de enfermagem. A importância deste grupo de estudo reside na possibilidade de uma maior integração dos envolvidos na assistência, resultando num cuidado autêntico e holístico.

$\mathrm{Na}$ categoria "Conhecer/compreender como as famílias percebem determinadas situações" (8,7\%) foram incluídos estudos que enfocam a motivação, o ambiente, as interações familiares e o significado da doença para a família. Acreditamos serem estes aspectos imprescindíveis, quando se pretende proporcionar ao ser humano doente, a assistência integrada e de qualidade, pois a presença da família é extremamente importante no processo terapêutico - promoção, manutenção, recuperação e reabilitação da saúde.

A seguir, encontramos as categorias "Propor/ construir/desenhar modelos teóricos" (8,7\%) voltadas para o processo de cuidar e ser cuidado, vivenciados no ambiente familiar; e "Conhecer como os integrantes da equipe de saúde atuam junto à família” (6,5\%), numa tentativa explícita de divulgar aspectos positivos da relação entre equipe e família e, ao mesmo tempo, identificar as lacunas e barreiras que possam interferir desfavoravelmente nesta relação. Finalmente, na categoria "Como os integrantes da equipe percebem a situação da família" (5,5\%), é possível constatar uma preocupação dos autores com o significado que a equipe profissional sustenta e valoriza na organização e implementação do 
cuidado em cada situação e ambiente específico.

O conjunto dos objetivos dos estudos em questão demonstra a preocupação da enfermagem brasileira em conhecer e compreender o contexto familiar principalmente em situações de doença - associado à necessidade de construir e implementar estratégias e modelos teóricos de assistência. Isto não difere do que tem constituído o foco da investigação da enfermagem familiar em outras culturas, em especial na norte-americana. A forma como a experiência de doença é vivenciada pela família, por exemplo, tem sido investigada em vários estudos, embora seja percebida e abordada de diferentes maneiras: "A família e a doença", "Resposta da família diante da doença" e "Impacto da doença sobre a família"(16).

\section{CONSIDERAÇÕES FINAIS}

Considerando que os trabalhos acadêmicos com família foram desenvolvidos, em grande parte, em um dos programas de pós-graduação da Região Sul, e que esses estudos guardam relação com algumas características identificadas nos resumos dos estudos de pós-graduação realizados em todos os pontos do país, acreditamos que, ao analisarmos uma parcela significativa da produção de enfermagem nesta área, estaremos, de certa forma, analisando uma boa parte da produção científica da enfermagem brasileira sobre família, muito embora não possamos fazer generalizações.

Os resultados revelam aumento do interesse em estudos com família por parte da Enfermagem a partir da década de 1990. Revelam também que muitos estudos ainda se encontram na fase exploratório-descritiva, com ênfase na compreensão da família e sua relação com a saúde e doença. Em relação ao estado da arte e perspectivas da investigação da enfermagem familiar, os resultados demonstram, mediante o grande número de estudos de intervenção caracterizados como pesquisa prática, um perfil diferenciado nos estudos de Enfermagem na Região Sul. Essa metodologia merece um olhar mais aprofundado em estudos futuros. Demonstram igualmente os resultados que a temática das investigações, embora diversa, gira em tomo do propósito de conhecer como a família cuida ou é cuidada e as implicações decorrentes desse fenômeno, e que os aspectos abordados emergem do cotidiano da enfermagem, apontando convergência entre a teoria e a prática.

O fato de os estudos se apresentarem quase sempre com objetivos amplos, assim como as metodologias adotadas, revelam o atual estado da arte da pesquisa com famílias pela Enfermagem, o que, sem dúvida, constitui reflexo do que ocorre também em outras áreas. Isto é compreensível, pois o trabalho com famílias quase sempre é focalizado sob a ótica de vários pesquisadores e a partir de suas práticas.

Outro aspecto importante observado foi o local onde os estudos foram desenvolvidos, porquanto, embora a maioria dos estudos tenha sido realizada no domićlio, uma parte deles demonstrou que tanto investigação como intervenção na família podem ocorrer em outros cenários, tais como o ambiente hospitalar (unidade de internação ou ambulatorial), o da unidade de saúde ou os espaços próprios da comunidade. Isso é muito importante, pois até o então, para nós, sempre prevaleceu a idéia de que a assistência à família só ocorria verdadeiramente quando feita fora do ambiente hospitalar, por exemplo. Agora, depois deste estudo, percebemos que assistir a família é possível em qualquer cenário, desde que os profissionais tenham um referencial teórico para direcionar seu agir, visto ser ele que fornece as ferramentas necessárias para a atuação do profissional neste novo enfoque da assistência.

Cumpre reconhecer que trabalhar com famílias se mostra hoje aos profissionais das mais diferentes áreas, como a "saída" possível para que, a médio e longo prazos, possam ser alcançados melhores resultados na qualidade da assistência e, por conseguinte, na condição de vida das famílias. Aos profissionais da área da saúde, portanto, de buscarem atualizar seus conhecimentos e, ao mesmo tempo, instrumentalizarem-se para assistir adequadamente a este " novo" objeto da assistência: a família que necessita de cuidados e/ou de conhecimentos e suporte para cuidar de seus membros e, desta forma, alcançar o viver-serestar-saudável em um mundo em transformação.

Estudos como opresente, permitem conhecer características importantes do que tem sido produzido no campo de estudo da Enfermagem brasileira com família. Consideramos que o trabalho que vem sendo desenvolvido é de grande valia, pois vem construindo um caminho próprio e uma história na pesquisa, e ambos podem contribuir no aprimoramento do conhecimento da ciência da família e da enfermagem familiar, cujo enfoque está no processo de viver em interface com a saúde e a doença e no encontro entre o cuidado profissional e familial.

Essa contribuição, no entanto, depende da divulgação que fizermos de nossos estudos. Da mesma forma que não podemos deixar de considerar o que tem sido produzido pela Enfermagem norte-americana, precisamos também reconhecer e valorizar o que temos produzido e, o mais importante, darmos a conhecer esse produto.

\section{REFERÊNCIAS}

1. Leininger M. Culture care diversity and universality: a theory of nursing. New York: National League for Nursing Press; 1991.

2. Marcon SS, Carreira L. Produccion cientifica de la 
enfermeria familiar Brasilena en el area de la família [abstract]. In: Conferencia Internacional de Enfermeria Familiar, 4, 1997. Valdivia, Chile. Program and Abstract, 1997. v. 1. p. 138 .

3. Marcon SS, Radovanovic CAT, Areas MMM. A Família como objeto de pesquisa da enfermagem brasileira. In: Mancia JR (org.). A enfermagem construindo sua agenda: entrelaçando a assistência, o ensino e a pesquisa. Brasília: Aben; 2003. p. 41-8.

4. Conselho Nacional de Saúde (Brasil). Resolução no 196, de 10 de outubro de 1996. Aprova diretrizes e normas regulamentadoras de pesquisas envolvendo seres humanos. Diário Oficial da União, Brasília, n.201, p.21082, 16 out. 1996 , seção I.

5. Minayo MCS. O desafio do conhecimento: pesquisa qualitativa em saúde. 4.ed. São Paulo: Hucitec Abrasco; 1996.

6. Gillis CL. Family research in nursing. In: Gillis CL, Highley BL, Roberts BM, Martinson IM. (Eds.) Toward a science of family nursing. Menlo Park, California: Addison-Wesley; 1989. p. 37-63.

7. Moriarty HJ. Key issues in the family research process: strategies for nurse researchers. ANS Adv Nurs Sci. 1990; 12(3):1-14. Review.

8. Waidman MAP, Marcon SS, Radovanovic CAT, Silva DMPP. Promover a vida: uma modalidade de cuidado à saúde na família e na comunidade. Texto \& Contexto Enfermagem. 2003; 12(3):332-41.

9. Elsen I, Althoff CR. Família e pesquisa: os contornos de um universo a explorar. In: Althoff CR, Elsen I, Nitschke RG, organizadores. Pesquisando a família: olhares contemporâneos. Florianópolis: Papa-Livro; 2004. p. 19-28.

10. McGoldrick M. Etnicidade e o ciclo de vida familiar. In: Carter B, McGoldrick M. As mudanças no ciclo de vida familiar: uma estrutura para a terapia familiar. $2^{a}$ ed. Porto Alegre: Artes Médicas; 1995. p. 65-83.

11. Gomes JV. Socialização primária: tarefa familiar? Cad Pesqui. 1994; (91): 54-61.

12. Travelbee J. Intervencion en enfermeria psiquiátrica: el processo de la persona a persona. Colombia: Oms; 1979.

13. Luz AMH, Mancia JR, Motta MGC, organizadores. As amarrras da violência - a família, as instituições e a Enfermagem. Brasília: Aben; 2004. p. 22-34.

14. Pena PA de. La investigación en enfermería familiar: una revision crítica. Avances en enfermeria 1994; $12(2 / 3): 38-46$.

15. Althoff CR, Shmickler CM, Mioto RCT, Manfrini GC. Pesquisando a família: por onde caminham os pesquisadores. In: Althoff CR, Elsen I, Nitschke RG, organizadores. Pesquisando a família: olhares contemporâneos. Florianópolis: Papa livro; 2004. p. 29-42.

16. Wall AL, Loveland-Cherry CJ. Family unit focused research: 1984-1991. In: Fitzpatrick JJ, Stevenson JS, editors. Annual review of nursing research. New York: Springer ; c1993. v.11. 